\title{
Tolerability of Duloxetine in the Elderly and in Young Adults: A Protocol and Preliminary Results of a Systematic Review and Individual Participant Data Meta-Analysis of Randomised Placebo-Controlled Trials.
}

Jean-Charles Roy ( $\square$ jean-charles.ROY@chu-rennes.fr)

Universite de Rennes 1 Faculte de Medecine https://orcid.org/0000-0003-0686-4099

Chloé Rousseau

Clinical Investigation Center, Rennes University Hospital

Alexis Jutel

University of Rennes 1 - Health Sciences Campus Villejean: Universite de Rennes 1 - Campus Sante de Villejean

Florian Naudet

Clinical Investigation Center, Rennes University Hospital

\section{Gabriel Robert}

University of Rennes 1 - Health Sciences Campus Villejean: Universite de Rennes 1 - Campus Sante de Villejean

\section{Protocol}

Keywords: Psychiatry, Geriatric, Meta-analysis, Drug Safety, Duloxetine Hydrochloride

Posted Date: January 20th, 2021

DOl: https://doi.org/10.21203/rs.3.rs-148334/v1

License: (c) (i) This work is licensed under a Creative Commons Attribution 4.0 International License.

Read Full License 


\section{Abstract}

\section{Background}

Duloxetine is an antidepressant that benefits from a wide range of approval in the elderly population, while its safety for use in the elderly population, compared to younger adults, is not clearly assessed. This protocol outlines a systematic review and individual participant data meta-analysis comparing the tolerability of duloxetine between elderly and younger adults.

\section{Methods}

Only randomised controlled clinical trials, comparing duloxetine to placebo, will be included in this metaanalysis. The studies will be selected if participants were adults of less and more than 65 years old, in conditions of use of duloxetine approved by the European Medical Agency (EMA) and the Food and Drug Administration (FDA). The primary outcome will be the rate ratio of serious adverse events under duloxetine compared to placebo, between participants at least 65 years old and younger adults. Second, the number of any adverse events, clinical efficacy and quality of life will be compared between elderly and younger adults under both interventions. The quality of evidence in the tolerability of duloxetine will be assessed using the GRADE system. A two-step random effect meta-analysis will be conducted. Preliminary searches and formal screening of search results against eligibility criteria on have been completed using predefined search term on electronic databases.

\section{Discussion}

This study represents the first meta-analysis investigating the safety of duloxetine in the elderly population across all conditions approved by European and American regulatory authorities. The results from this meta-analysis are intended to help prescribers to provide better care for the elderly population.

Systematic review registration

The protocol has been registered at the International Prospective Register of Systematic Reviews (PROSPERO; registration number: CRD42019130488).

\section{Background}

\section{Rationale}

Duloxetine is a medication that has been approved for a wide range of pathologies by the Food and Drug Administration (FDA) and the European Medicines Agency (EMA), namely, major depressive disorder, generalized anxiety disorder, diabetic peripheral neuropathic pain, fibromyalgia, chronic musculoskeletal pain and stress urinary incontinence. These conditions represent a major public health burden for the elderly population ${ }^{1}$. While several guidelines recommend the use of duloxetine as a first-line medication for these conditions in the elderly ${ }^{2-6}$, others formally proscribe it ${ }^{7}$. In fact, good practice guidelines for 
duloxetine use are under debate, notably because of mixed evidence for a favourable risk-benefit balance in older populations $s^{5,8}$.

For instance, a mixed treatment meta-analysis, mostly based on indirect evidence, suggests that duloxetine had higher response rates than other antidepressants for major depressive disorder in the oldage population ( $\geq 65$ years old), in the context of scarce data in this population ${ }^{9}$. In contrast, duloxetine was found to increase the risk of adverse events ${ }^{10}$. In younger adults, several meta-analyses concluded that duloxetine was associated with a disfavouring risk-benefit balance compared to placebo in osteoarthritis and stress urinary incontinence ${ }^{11,12}$. Meta-regression analyses from Cipriani et al. (2018) found similar results, observing a significant association between the mean age of participants and response to duloxetine (odds ratio $(O R)=1.86$, confidence interval $(\mathrm{Cl}) 95 \%=1.66-2.08$ ) with an association between age and number of drop-outs under duloxetine $(\mathrm{OR}=1.10, \mathrm{Cl} 95 \%=0.97-1.24)^{13}$, although the latter result did not reach statistical significance.

Concerns about duloxetine use in the elderly have existed since premarketing pharmacokinetic studies. Study SAAY concluded a reduced oral clearance of duloxetine of $25 \%$ in an elderly class (mean age of 69 years old) compared to a middle-aged class (mean age of 42 years old) ${ }^{14}$ of women under $40 \mathrm{mg}$ of duloxetine. Furthermore, the area under the curve measure of exposure was found to be $24 \%$ higher in elderly individuals, although it was not statistically significant in the cohort of 24 participants, but with half-life elimination reduced by one-third in elderly individuals compared to that in middle-aged individuals. A statistically significant correlation of diminished oral clearance of duloxetine with age was demonstrated, which was estimated to be a reduction in clearance of almost $60 \%$ in individuals between 23 and 76.8 years old (Study SAAB) ${ }^{14}$. Skinner et al. (2004) estimated that a decrease in oral clearance accelerates at approximately 52 years of age $\mathrm{e}^{15}$. Similar results have been reproduced in ulterior pooled analyses of phase III trials ${ }^{16}$ with comparable effect sizes (the EMEA/H/C/572/II/26 procedure reported a $25 \%$ decrease in oral clearance in individuals between 29 and 69 years old). Taken together, these early phase I studies demonstrated increased systemic exposure to duloxetine in the elderly population.

However, the clinical interpretation of pharmacokinetic results remains challenging. Many subgroup analyses of pilot trials did not find quantitative differences in tolerability between old and younger adults. One plausible explanation lies in the small sample sizes of elderly participants included in these studies $^{16}$. As mentioned in the FDA CYMBALTAC Label $^{17}$ (revised version of 2017), of the 6781 patients in premarketing clinical studies of duloxetine in all conditions, $15.6 \%$ were aged 65 years or over. Although it represents approximately 1058 subjects, no direct comparison of between-age tolerability across conditions has been found in the literature. Altogether, it seems that the tolerability of duloxetine in elderly individuals needs clarification.

Therefore, we planned to determine whether the use of duloxetine in elderly adults is associated with a higher risk of both serious adverse events (SAEs) and non-serious adverse events (nsAEs) than those in 
younger adults. We therefore designed an individual participant meta-analysis of randomized controlled trials (RCTs) of any condition with FDA or EMA approval of the use of duloxetine.

\section{Objectives}

Our main objective is to compare the incidence rates of SAEs under duloxetine in comparison to placebo between participants aged 65 or older and younger adults between 18 and 65 years old, in RCTs for EMA and FDA approved conditions. Our primary hypothesis is that the elderly population has higher incidence rates of SAEs caused by duloxetine than younger adults.

Our secondary objectives are to compare the incidence rates of nsAEs caused by duloxetine between participants at least 65 years of age and younger adults, and to compare both the clinical efficacy of duloxetine on clinical scales and quality of life between elderly individuals and younger adults separately for each indication. Furthermore, efficacy and tolerability will be compared between younger elderly (aged between 65 and 75 years) and older elderly (aged 75 years or older) participants.

\section{Methods}

This systematic review and meta-analysis of individual participant data (IPD) will be conducted in interventional, prospective, double-blind, randomized, placebo-controlled trials with an independent duloxetine arm. According to a protocol registered on PROSPERO (systematic review registration-2019 CRD42019130488), we conducted a systematic literature review of relevant trials performed on adult participants aged $<$ and $\geq 65$ years old in a population suffering from a condition having EMA or FDA approval for duloxetine. In this report, we describe all eligible studies and present the outcomes that will be extracted for the IPD and the methods that will be used for data synthesis following the PRISMA-P recommendations (Additional file 1). The anticipated end date of the study is November 2022.

\section{Eligibility criteria}

We used the following eligibility criteria:

- Types of studies: Double-blind RCTs in adult population with both adults ( $\geq 18$ year old) participants $\geq$ and $<65$ years of age;

- Types of participants: subjects suffering from a disorder with a known approval for duloxetine by the FDA and the EMA, namely, depression, anxiety, diabetic neuropathic pain, fibromyalgia, chronic musculoskeletal pain and stress urinary incontinence;

- Types of interventions: duloxetine regardless of the dosage, administration frequency, and route of administration;

- Type of comparator: placebo;

- Types of outcome measures: report of SAEs for each participant under duloxetine and placebo arms and/or nsAEs and/or efficacy and/or quality of life. 
The research was restricted to trials written in English, regardless of their publication status (published/unpublished).

\section{Information sources}

Searches were conducted in PubMed (to identify individual studies from published systematic reviews and meta-analyses of duloxetine), ClinicalTrials.gov, Clinicaltrialsregister.eu, data sharing platforms (ClinicalStudyDataRequest.com, YODA and Vivli), FDA drug approval packages and European public assessment reports and withdrawn applications from the EMA website. The review was performed on studies available in electronic databases from their date of inception to 31 May 2019.

\section{Search strategy}

Trial identification was systematic with different search strategies depending on the source. First, we searched PubMed for all systematic reviews and meta-analyses involving duloxetine for an approved indication. Then, individual trials were identified from these systematic reviews and meta-analyses. The search terms were as follows: "(Duloxetine AND Meta-Analysis[ptyp])". In ClinicalTrials.gov, the search was restricted to all interventional studies with adults and older adults, using "duloxetine" as the search term. In Clinicaltrialsregister.eu and in data sharing platforms, the search term was "duloxetine" with no filter applied. On the FDA website, FDA drug approval packages were downloaded from the FDA approved drug product, entering "duloxetine" as the search term. On the EMA website, European public assessment reports and withdrawn applications were selected, which were limited to reports on humans with no restriction on the authorization status and using "duloxetine" as the search term. The list of included studies as the list of meta-analyses and systematic reviews are reported in additional file 2 and additional file 3 , respectively.

\section{Study records}

\section{Data management, selection and collection processes}

Selection and coding of the different study characteristics were performed by two independent reviewers (JCR and AJ) in a blinded manner. A third reviewer (FN) arbitrated in case of disagreement. Studies appearing to duplicate authors, treatment comparisons, sample sizes and outcomes were checked one against another to avoid double-counting and integrating data from several reports on the same study and in contact with the study sponsors. A data extraction sheet based on the Cochrane Handbook for Systematic Reviews of Interventions guidelines was developed. In case of missing data, the sponsor of the study and/or corresponding authors were contacted.

\section{Collecting IPD}

A data sharing request was sent to all sponsors for which trials were spontaneously available on data sharing platforms. For the remaining studies, the request was sent to all corresponding authors, and if possible, a research proposal was addressed to each pharmaceutical sponsor on data sharing platforms (for Eli Lilly, Shionogi, Pfizer and AbbVie trials) or on the sponsor website (for Lundbeck, Takeda and 
Merck Sharp \& Dohme trials). For willing collaborators, the terms of the collaboration will be specified in a data transfer agreement, signed by representatives of the data provider and of the recipients (Clinical Investigation Center, Department of Clinical Pharmacology, Rennes University Hospital, France). The collection of IPD is ongoing.

The requested participant characteristics are baseline age, gender, intervention arm, duloxetine dose, duration of treatment, number of SAEs from baseline to endpoint, number of nsAEs from baseline to endpoint, study primary outcome and its values at baseline and at endpoint, type of quality of life scale used and its values at baseline and at endpoint. Data will be accepted in any suitable electronic format. Checks on the data will be made to ensure data are correctly coded, missing data are correctly identified, extreme values are genuine and data are consistent with published results. Data from all trials will be incorporated into a single database with fields that are consistent across trials.

\section{Data items}

For each included study, information will be extracted on:

- Characteristics of the study: year, country, number of arms with duloxetine and placebo, funding sources, conflict of interest, and condition;

- Characteristics of trial participants: mean age (and its standard deviation), gender, number of patients included in the analysis, and population of analysis used in the identified report (intention to treat, per protocol, other);

- Type of administration, dose and duration;

- Outcome measures as stated above (including the exact definition of outcome (e.g., MedDRA or other)).

\section{Outcomes and prioritization}

The main outcome is the number (count) of SAEs for each individual patient. In our study, SAE refers exclusively to any undesirable experience associated with the use of a medical product that results in death, is life-threatening, requires hospitalization or prolongation of existing hospitalization, results in persistent or significant disability, or is a congenital anomaly or birth defect. This outcome has the advantages of being simple to interpret and clinically relevant for safety estimation, containing a severity criterion from its definition.

Additionally, the following secondary outcomes will be assessed:

- The number of nsAEs: nsAE refers to any untoward medical occurrence in a clinical trial subject administered a medicinal product that does not have severity criteria for a SAE or necessarily has a causal relationship with this treatment. In a randomized placebo-controlled setting, this outcome provides a global estimate of treatment safety, without the severity feature of the SAE. 
- The efficacy on the clinical scale for each indication (i.e., depression, anxiety, pain, and urinary incontinence): different scales with the same indication will be standardized by z-scores. This outcome will evaluate the clinical benefit in patients.

- The quality of life scores for each indication: the different scales will be standardized by z-scores. In studies where multiple quality of life scales were used, a hierarchy was established by selecting scales that both resume health data in one unique total score and that were the most used among the duloxetine trials. If the only available instrument in a trial did not permit us to resume data into one score (e.g., SF36), its general health subscale was retained as an indicator of quality of life (detailed in Additional file 4 and Table S1). Quality of life scores yield a broad and ecological estimate of the well-being state of the patient.

\section{Risk of bias in individual studies}

Two researchers (JCR and AJ) will assess each trial for risk of bias independently, addressing randomization, allocation concealment, blinding of assessors and of study participants, completeness of outcome assessment, selective reporting and other potential sources of bias according to the Cochrane Collaboration tool for assessing risk of bias in its current version, RoB2 ${ }^{18}$, at the study level. Discrepancies will be resolved by consensus.

\section{Data synthesis \\ Main analysis}

As all data are not directly downloadable together but rather provided remotely on separate interfaces, we chose a two-step procedure ${ }^{19}$ to derive the incidence rate ratio (for counts of binary outcomes) and mean differences (for quantitative outcomes).

The first step consists of comparing the number (count) of SAEs between groups of participants arranged by age using a generalized linear mixed model (with a quasi-Poisson link function) in each individual study. Age (binary variable between "young" participants between 18 and 65 years old, and "old" participants $\geq 65$ years old) and intervention (binary variable between participants under duloxetine and participants under placebo) will be considered fixed effects, and their interaction will be explored as the main comparison. The incidence rate ratios, as well as their variances, will be extracted from the interaction between age and intervention.

In the second step, we pooled the extracted incidence rate ratios using a random effect meta-analysis. Similarly, the number of nsAEs (count) will be analysed using a two-step approach based on a generalized linear mixed model (with a quasi-Poisson link function). For both SAEs and nsAEs, we will use meta-regressions to explore whether these interactions between age and intervention are different across the different conditions (major depressive disorder, generalized anxiety disorder, diabetic peripheral neuropathic pain, fibromyalgia, chronic musculoskeletal pain and stress urinary incontinence). 
Summary measures for the analyses of efficacy and quality of life: in each condition, clinical scales (i.e., depression, pain, and chronic anxiety) and quality of life scales will be analysed separately using a similar two-step approach relying on the results of a linear model. As various scales might be used across studies, we will use z-scores to standardize the different scales and populations.

Missing data will be handled by multiple imputation.

\section{Sensitivity and subgroup analyses}

A sensitivity analysis will be performed considering the occurrence of SAEs in binary (Yes vs. No) with the same two-step approach relying on a generalized linear mixed model (with a logistic link function) in each individual study.

A subgroup analysis will be performed applying the same methodology as for adverse events to explore differences between younger elderly (between 65 and 74 years old) and older elderly participants ( $\geq$ 75 years old) in terms of adverse events (serious and non-serious) and efficacy.

\section{Meta-biases}

Missing trials will be explicitly reported with their characteristics and results described. Duplicate publication bias will be limited by the selection and precise identification of published and unpublished trials. If one or more trials could not be precisely identified by at least an ID number, these trials will be reported in the study results with explained attempts to obtain identification.

Countries where studies took place will be systematically assessed in the description studies table to account for possible location bias. In addition, we clearly outline in our methods that our analysis selected only studies written in English.

\section{Confidence in cumulative evidence}

We will use GRADE to rate the overall certainty (quality) of evidence, which includes the evaluation of risk of bias, inconsistency, indirectness, imprecision and publication factors ${ }^{20}$.

\section{Discussion}

To our knowledge, this study represents the first meta-analysis investigating the safety and utility of duloxetine in the elderly population across all conditions approved by European and American regulatory authorities. The scope of our meta-analysis is intended to benefit a wide range of medical specialties to help prescribers and to provide better care for the elderly population. Furthermore, our study exemplifies the current possibilities and limits of sharing individual data in meta-research.

\section{Abbreviations}

Cl: confidence interval 
EMA: European Medical Agency

FDA: Food and Drug Administration

IPD: individual participant data

nsAE: non-serious adverse event

OR: odds ratio

RCT: randomized controlled trial

SAE: serious adverse event

SF36: 36-Item Short Form Survey

\section{Declarations}

\section{Ethical Approval and Consent to participate}

Not applicable.

\section{Consent for publication}

Not applicable.

\section{Availability of data and materials}

We will publish the codes in the supplementary material of the systematic review. No individual participant data will be published as contracted with the data sharing platform.

\section{Competing interests}

The authors declare that they have no competing interests.

\section{Funding}

There is no external funding for this review.

\section{Authors' contributions}

JCR wrote up the protocol with supervision from FN and GR. FN, CR and JCR wrote the 'Methods' section. FN contributed with expert knowledge on pharmacology. GR contributed with expert knowledge on geriatrics psychiatry. All authors read and approved the final manuscript. 
Not applicable.

\section{Authors' information}

Adult Psychiatry Department, Rennes University Hospital, Rennes, France.

Jean-Charles Roy, Alexis Jutel, Florian Naudet \& Gabriel Robert

Clinical Investigation Center INSERM 1414, Department of Clinical Pharmacology, Rennes University Hospital, Rennes, France.

Chloé Rousseau \& Florian Naudet

\section{References}

Citation list not provided with this version. Additional files 2 and 3 contain lists of eligible studies.

\section{Supplementary Files}

This is a list of supplementary files associated with this preprint. Click to download.

- Additionalfile1.docx

- Additionalfile2.docx

- Aditionalfile3.docx

- Additionalfile4.docx 